\title{
VALIDACIÓN DE MATERIALES DIDÁCTICOS SOBRE ALIMENTACIÓN SALUDABLE EN EL SUR DE YUCATÁN.
}

\author{
VALIDATION OF EDUCATIONAL MATERIALS ON HEALTHY EATING IN SOUTHERN YUCATÁN.
Canul Tamay Neysi María, Barradas Castillo María del Rosario, Lendechy Grajales Ángel Cirilo, Pavía Carrillo Emilio Felipe.

Universidad Autónoma de Yucatán: 1 Facultad de Medicina, 2 Centro de Investigaciones Regionales "Dr. Hideyo Noguchi". México.

\section{RESUMEN}

Introducción: El cambio en la selección de alimentos, preferentemente de tipo industrializados y/o hipercalóricos, contribuye en los problemas de salud. De aquí la importancia de implementar materiales didácticos validados como una estrategia para la promoción de la salud. Objetivo: Validar dos materiales didácticos en la población sur de Yucatán para la mejora en la promoción de la alimentación saludable. Material y Método: Estudio transversal mixto, con muestra a conveniencia de 112 personas de 18-60 años. La investigación se llevó en las siguientes etapas: Preproducción, Elaboración y Validación, esta última se dividió en tres fases: 1 . Validación técnica, con la participación de nutriólogos; 2 . Prueba piloto, realizada con personas con características similares a la población objetivo y 3 . Validación con la población. Las variables estudiadas fueron: atractividad, comprensión, identificación, aceptación e inducción a la acción. Se aplicó el coeficiente de alfa de Cronbach para evaluar la confiabilidad y validez del instrumento. Resultados: El rotafolio reflejó una confiabilidad aceptable $(\alpha=0.761)$ y el franelógrafo una confiabilidad buena $(\alpha=0.832$ ). las variables con mayor necesidad de mejoras fueron: comprensión, aceptación e identificación en ambos materiales didácticos. Conclusiones: Los materiales son estadísticamente confiables, aunque son susceptibles de mejorarlos atendiendo las variables, comprensión, aceptación e identificación.

Palabras Clave: Validación, Materiales didácticos, Alimentación saludable.

\section{ABSTRACT}

Introduction: The change in the selection of foods, preferably industrialized and / or hypercaloric, contributes to health problems. Hence the importance of implementing validated teaching materials as a strategy for health promotion. Objective: "To validate two teaching materials in the southern population of Yucatán to improve the promotion of healthy eating." Material and method: Mixed cross-sectional study, with a convenience sample of 112 people aged 18-60 years. The research was carried out in the following stages: Preproduction, Elaboration and Validation, the latter was divided into three phases: 1. Technical validation, with the participation of nutritionists; 2 . Pilot test, carried out with people with characteristics similar to the target population and 3. Validation with the target population. The variables studied were: attractiveness, understanding, identification, acceptance and induction to action. Cronbach's alpha coefficient was applied to assess the reliability and validity of the instrument. Results: The flip chart showed acceptable reliability $(\alpha=0.761)$ and the flannel board a good reliability $(\alpha=0.832$ ). the variables with the greatest need for improvement were: understanding, acceptance and identification in both teaching materials. Conclusions: The materials are statistically reliable, although they can be improved by taking into account the variables, understanding, acceptance and identification.

Key words: Validation, Didactic materials, Healthy eating.

Correspondencia: Neysi María Canul Tamay A17016971@alumnos.uady.mx

Recibido: 05 de octubre 2021, aceptado: 18 de noviembre 2021

(C) Autor2022

\section{(c) (1)}

DOI: https://doi.org/10.29105/respyn21.1-3

Citation: Canul Tamay N.M., Barradas Castillo M.R., Lendechy Grajales A.C., Pavía Carrillo E.F. (2022)

Validación de materiales didácticos sobre alimentación saludable en el sur de Yucatán. Revista Salud

Pública y Nutrición, 21 (1), 19-27. 


\section{Artículo Original}

\section{Introducción}

En las últimas décadas la modernización, favorecida por la urbanización y las nuevas tecnologías, ha traído comodidad y en ciertos aspectos una mejora en la calidad de vida de nuestra sociedad; sin embargo, estos cambios han afectado $\mathrm{y}$ modificado drásticamente el estilo de vida y la alimentación de la poblacional mexicana (Ayuso, y Castillo, 2017).En la actualidad es muy notorio y preocupante cómo estos cambios han provocado que la dieta tradicional de las comunidades rurales, basadas principalmente en alimentos frescos y naturales, cultivados por ello mismos, como es el maíz, frijol y chile, se vea sustituidos por la dieta occidental, dominado principalmente por los productos industrializados, alimentos hipercalóricos, abundantemente en azúcares simples y grasas saturadas (Marín, Sánchez y Maza, 2014). Esta transición alimentaria ha favorecido problemas de salud de la población, reflejándose en el aumento de la prevalencia e incidencia de sobrepeso y obesidad, así como de las enfermedades crónico-degenerativas. Este panorama de salud emergente a nivel mundial hace un llamado para involucrarse en la Promoción de la Salud, la cual abarca un conjunto de intervenciones y acciones que van dirigidas a beneficiar y proteger el estado de salud, así como la calidad de vida, mediante la prevención y solución de causas primordiales de los problemas (OMS,2017), siendo por lo tanto una vía indispensable para persuadir a la sociedad hacia hábitos y prácticas alimentarias saludables. Por lo anterior, en la educación para la salud, considerado el instrumento de la promoción, es sumamente necesario el empleo de materiales didácticos para facilitar la transmisión del mensaje a la población y ayudar a construir conocimiento, así como favorecer el proceso de enseñanza aprendizaje (Díaz, Pérez, Báez y Conde, 2012) y (Vásquez, 2014). Sin embargo, el impacto que tendrán los materiales didácticos depende en gran medida de su diseño y contenido, así como de su correcta adaptación a las características y necesidades de los destinatarios (Salazar, Shamah, Escalante, Jiménez, 2012). Consciente de esto, la Organización Panamericana de la Salud (OPS, 1984) recalca la necesidad del uso de materiales didácticos sencillos, de calidad y acorde a las características de la población para promover el autocuidado y las medidas preventivas en salud.
Se han establecido dos tipos de validación, la primera, es la técnica; su finalidad es asegurar que los contenidos sean claros y apropiados, mediante una revisión efectuada por un grupo de expertos en el tema que se aborda. La segunda es la validación con la población, la cual requiere de dos grupos; el piloto y el objetivo (UNICEF,2003). El grupo piloto, debe tener características similares a la población de interés, con el fin de identificar la mejora en el contenido, el lenguaje, así como, conocer si la estructura y diseño del material funciona y es apto para ser utilizado, para que, finalmente la validación se realice con la población objetivo.

Existen autores que ratifican la importancia de la validación de materiales didácticos, "El proceso de validación es necesario antes de usar los materiales como ayuda educativa, con el fin de valorar su pertinencia, validez y potencialidad para motivar la adopción voluntaria de conductas saludables" (Arismendi, Carmona, Rodríguez y Alzate, 2015, p.67). De igual manera Franco, Alzate, Granda, Hincapié y Muñoz (2018), exponen la gran importancia de evaluar los materiales en el área de la salud, para lograr su adaptación y adecuación a las características y necesidades de la población y con esto poder garantizar la calidad y efectividad, evitando de esta manera el uso de material didáctico sin impacto educativo.

Por lo mencionado anteriormente se recalca la importancia de validar materiales, tomando en cuenta los criterios de Ziemendorff y Krause (2003): atractividad, comprensión, identificación, aceptación e inducción a la acción. La atractividad hace referencia a que el material contiene elementos suficientemente buenos para captar y llamar la atención; la evaluación se da mediante aspectos como los colores, las imágenes, el tipo de letra o diseño que se está utilizando (UNICEF,2003). El segundo (comprensión), evalúa si la población entiende y tienen claro el mensaje o si hay existencia de confusión respecto al contenido del material. El tercero (identificación), tiene en cuenta lo sociocultural, por lo que explora si la población se siente identificada con las ideas y el contenido plasmado, evidenciando si perciben que el material fue elaborado y dirigido específicamente para ellos (Franco, et al.,2018). El cuarto (aceptación), su evaluación da como resultado conocer si hay indignación con el mensaje, el lenguaje, las 
imágenes, el enfoque dado y el contenido; en caso contrario a esto, evidencia la aceptación de la población respecto a las características del material, las ideas, así como las propuestas o acciones que se quieren alcanzar. El último (inducción a la acción), explora si se percibe que el contenido del material va a favorecer cambio en el comportamiento, es decir, identificar si se favorece la motivación y adopción de los comportamientos dirigidos al mejoramiento de salud (Barradas, Lendechy, Coop y Pavía, 2021).

De aquí la relevancia de la validación de los materiales didácticos, porque permite identificar los aspectos de mejora y lograr focalizar los materiales al grupo de interés, para favorecer la adecuada transmisión del mensaje. Por lo contrario el omitir este proceso de validación solo propicia caer en el error de contemplar solo el punto de vista, las exigencias de las personas involucradas en la elaboración, así como el no considerar las diferencias que existen, en el aspecto socioeconómico, sociocultural, el nivel educativo y las perspectivas de la población objetivo, dando como resultado que se siga invirtiendo en la elaboración de materiales didácticos inadecuados, carentes en características necesarias para alcanzar su efectividad y calidad (Ziemendorff y Krause, 2003).

Por todo lo anterior, se tiene como objetivo "Validar dos materiales didácticos en la población sur de Yucatán para la mejora en la promoción de la alimentación saludable". Este estudio traerá beneficios tanto para la región sur como para la sociedad en general, ya que al contar con material didáctico validado de calidad y focalizado a la población, permitirá que haya una mejor comprensión y sobre todo eficacia en la transmisión de la información, lo que a su vez motive a la población a realizar acciones que beneficien su salud.

\section{Material y Método}

Se realizó un estudio transversal mixto, donde el universo fue la población adulta de las localidades de Tixméhuac, Tahdziú y Chacsinkín, con una muestra a conveniencia de hombres y mujeres adultos de 18 a 60 años, conformada por 112 personas; 12 participaron en la prueba piloto (cinco para el rotafolio y siete para el franelógrafo) y 100 en la validación de ambos materiales (50 para el rotafolio y 50 en el franelógrafo). Todos los participantes firmaron el consentimiento informado.
Se excluyeron aquellas que no tenían disponibilidad para participar en las reuniones programadas y quienes no cumplieron con los protocolos sanitarios. Previo a la validación de los materiales se llevó a cabo la validación técnica conformada por cuatro profesionales del área de Nutrición de la Universidad Autónoma de Yucatán (UADY).

Para el desarrollo de los instrumentos de validación se siguieron las recomendaciones de Ziemendorff y Krause (2003) y la OPS (1984), incluyéndose los siguientes criterios: atractividad, aceptación, comprensión, identificación e inducción a la acción. El instrumento se estructuró en 8 o 9 ítems para valorar cada una de las variables, haciendo un total de 40 ítems, con una escala de Likert del 1 al 5, donde 1 representa un menor complimiento y 5 un mayor cumplimiento (9). La escala para evaluar los resultados del instrumento fue: $40-45$ puntos no necesita reformas, 21-39 necesita reformas y $\leq 20$ puntos el material debe ser rechazado (OPS, 1984).

Para la captura de los resultados obtenidos, se elaboró la base de datos, donde se ingresó la información de cada etapa de validación; el análisis y tabulación se realizó mediante estadística descriptiva, con software SPSS®, versión 25 en español.

La evaluación de la confiabilidad del instrumento se realizó con el coeficiente alfa de Cronbach, la cual refleja la consistencia entre los diferentes ítems y si existe correlación entre sí (Chaves y Rodríguez, 2017). Los criterios de interpretación se muestran en la tabla 1 .

Tabla 1. Valoración de confiabilidad según el coeficiente alfa de Cronbach.

\begin{tabular}{cc}
\hline $\begin{array}{c}\text { Intervalo de coeficiencia de } \\
\text { Alfa de Cronbach }\end{array}$ & Interpretación \\
\hline $0-0.5$ & Inaceptable \\
$0.5-0.6$ & Pobre \\
$0.6-0.7$ & Cuestionable \\
$0.7-0.8$ & Aceptable \\
$0.8-0.9$ & Bueno \\
$0.9-0.95$ & Excelente \\
\hline
\end{tabular}

Fuente: Chaves y Rodríguez (2017). 
El proceso de elaboración de los materiales y su respectiva validación consistió en las siguientes etapas: La primera (preproducción) consistió en la búsqueda de información bibliográfica sobre alimentación saludable en la etapa adulta) y se estructuró la ficha técnica de la información. La segunda fue la elaboración de los materiales didácticos, la cual consistió en definir primero la secuencia didáctica y posteriormente la organización de la información en el material. La tercera fue la validación y consistió en tres fases; la técnica, la prueba piloto y la poblacional.

En la validación técnica, participaron cuatro profesionales del área de la nutrición de la UADY, a quienes se les presentó los materiales elaborados y se solicitó su evaluación mediante el instrumento de validación. Derivado de los puntajes y observaciones de los expertos se hicieron cambios en la primera versión de los materiales didácticos.

En la prueba piloto participaron 12 mujeres, de las cuales cinco valoraron el rotafolio y siete el franelógrafo, quienes cumplían con características similares a las personas que colaboraron en la validación poblacional. El proceso de validación dio inicio con la exposición del tema utilizando los materiales didácticos. Se realizaron dos sesiones de 45 min para cada material, al término de la presentación del tema se proporcionó a cada participante el instrumento de validación para que lo contestaran. Derivado de los puntajes y observaciones se hicieron cambios en los materiales. Cabe destacar que en ambas pruebas piloto la explicación del tema de ambos materiales se realizó en lengua maya, debido a que la mayor parte de los participantes refirió hablar y entenderla al ser su lengua materna.

La Validación poblacional, se llevó a cabo con la participación de un total de 50 personas por cada material didáctico, dando inicio con la exposición del tema, con una duración de 45 min para cada material (franelógrafo y rotafolio). Finalizando la exposición del tema se proporcionó el instrumento de validación para que los participantes evaluarán el material didáctico. Debido a la contingencia por Covid-19, fue necesario convocar a la población por grupos de 10 personas para guardar la sana distancia y aplicar las medidas sanitarias establecidas por el gobierno.
A continuación, en la figura 1, se esquematiza el proceso de validación de los materiales didácticos.

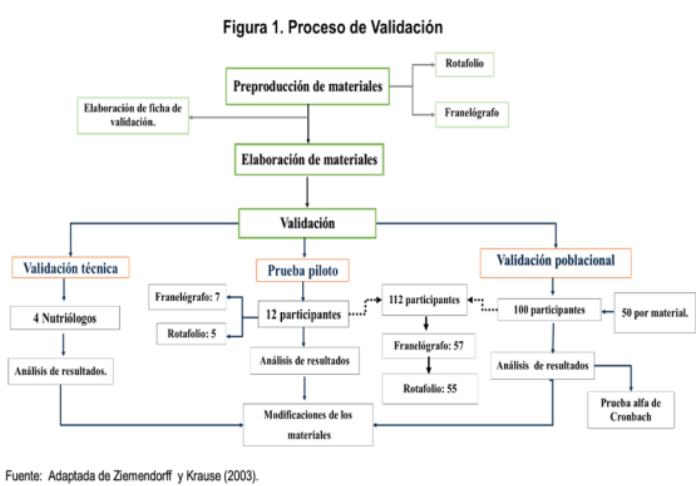

\section{Resultados}

En la investigación participaron 112 adultos, de los cuales el $70.5 \%$ eran mujeres y el $29.5 \%$ hombres. Del total de la muestra el $11 \%$ corresponde a las 12 personas que integraron las pruebas piloto; y el $89 \%$ corresponde a los 100 participantes de la validación poblacional. A continuación, se presentan los resultados de la investigación en el orden en que se realizó la validación:

Los resultados de la validación técnica del franelógrafo (Tabla 2) muestran que la atractividad e inducción a la acción no requieren reformas, mientras que las tres variables restantes sí muestran necesidad de reformas. Los cambios sugeridos por los expertos fueron: eliminar algunas imágenes utilizadas en el apartado de características de la alimentación saludable y la de las recomendaciones. Por su parte, en la validación técnica del rotafolio (Tabla 2), se observó que la atractividad e inducción a la acción reflejó no necesitar reformas, mientras que la comprensión, aceptación e identificación requieren reformas. Los cambios sugeridos por los expertos fueron: necesidad de eliminar palabras técnicas, mejorar la redacción, así como cambiar algunas imágenes que causan confusión en el apartado de las recomendaciones. 


\begin{tabular}{|c|c|c|c|c|c|c|}
\hline Validación & Clasificación & $\begin{array}{c}\text { Comprensión } \\
\mathrm{n}(\%)\end{array}$ & $\begin{array}{l}\text { ractividad } \\
n(\%)\end{array}$ & $\begin{array}{l}\text { ceptación } \\
\mathrm{n}(\%)\end{array}$ & $\begin{array}{l}\text { entificación } \\
\mathrm{n}(\%)\end{array}$ & $\begin{array}{c}\text { Inducción a } \\
\text { la acción } \\
n(\%)\end{array}$ \\
\hline \multirow{2}{*}{$\begin{array}{c}\text { Técnica } \\
\text { (Franelógrafo) }\end{array}$} & $\begin{array}{c}\text { No necesita } \\
\text { reformas }\end{array}$ & $2(50)$ & $4(100)$ & $2(50)$ & $3(75)$ & $4(100)$ \\
\hline & $\begin{array}{l}\text { Necesita } \\
\text { reformas }\end{array}$ & $2(50)$ & 0 (N/A) & $2(50)$ & $1(25)$ & $0(\mathrm{~N} / \mathrm{A})$ \\
\hline \multirow{2}{*}{$\begin{array}{c}\text { Técnica } \\
\text { (Rotafolio) }\end{array}$} & $\begin{array}{l}\text { No necesita } \\
\text { reformas }\end{array}$ & $3(75)$ & $4(100)$ & $2(50)$ & $3(75)$ & $4(100)$ \\
\hline & $\begin{array}{l}\text { Necesita } \\
\text { reformas }\end{array}$ & $1(25)$ & 0 (N/A) & $2(50)$ & $1(25)$ & $0(\mathrm{~N} / \mathrm{A})$ \\
\hline
\end{tabular}

N/A: No aplica, referente a 0 participantes.

Como se puede observar en la tabla 3 , en la prueba piloto las variables de atractividad, aceptación, identificación e inducción a la acción no necesitan reformas y solo la comprensión reflejó necesidad de reformas, con un porcentaje de $14 \%$, que corresponde a un participante. Las sugerencias de mejora fueron que se modificaran algunas palabras para la explicación del tema, ya que el lenguaje fue muy técnico.

Por otro lado, en la tabla 4 , se observa, que en la prueba piloto las variables de atractividad, identificación e inducción a la acción no reflejaron necesidad de reformas, siendo solo variables de comprensión y aceptación, los que presentaron necesidad de reformas, con porcentaje un $20 \%$, que equivalen a un participante en cada uno. Se identificó que los participantes consideran que el material contiene una amplia información, por lo cual refieren no comprender en concreto todo lo que se explicó, de igual manera, se identificó que algunos elementos del material no son considerados por los participantes como parte de su cultura, por lo tanto, no se sentían identificados.

\begin{tabular}{|c|c|c|c|c|c|c|}
\hline \multirow[b]{2}{*}{ Validación } & \multirow[b]{2}{*}{ Clasificación } & \multicolumn{2}{|c|}{ Comprensión Atractividad } & \multicolumn{2}{|c|}{ Aceptación Identificación } & \multirow{2}{*}{$\begin{array}{c}\begin{array}{c}\text { Inducción } \\
\text { a la acción }\end{array} \\
\mathrm{n}(\%) \\
\end{array}$} \\
\hline & & $n(\%)$ & $n(\%)$ & $n(\%)$ & $n(\%)$ & \\
\hline \multirow{2}{*}{$\begin{array}{l}\text { Prueba } \\
\text { piloto }\end{array}$} & $\begin{array}{c}\text { No necesita } \\
\text { reformas }\end{array}$ & $6(86)$ & $7(100)$ & $7(100)$ & $7(100)$ & $7(100)$ \\
\hline & $\begin{array}{l}\text { Necesita } \\
\text { reformas }\end{array}$ & $1(14)$ & $0(\mathrm{~N} / \mathrm{A})$ & $0(\mathrm{~N} / \mathrm{A})$ & $0(N / A)$ & $0(N / A)$ \\
\hline \multirow{2}{*}{ Poblacional } & $\begin{array}{l}\text { No necesita } \\
\text { reformas }\end{array}$ & $34(68)$ & $45(90)$ & $32(64)$ & $37(74)$ & $48(96)$ \\
\hline & $\begin{array}{l}\text { Necesita } \\
\text { reformas }\end{array}$ & $16(32)$ & $5(10)$ & $18(36)$ & $13(26)$ & $2(4)$ \\
\hline
\end{tabular}
N/A: No aplica, referente a 0 participantes.

\begin{tabular}{|c|c|c|c|c|c|c|}
\hline Validación & Clasificación & $\begin{array}{c}\text { Comprensión } \\
n(\%)\end{array}$ & $\begin{array}{c}\text { Atractividad } \\
\mathrm{n}(\%) \\
\end{array}$ & $\begin{array}{c}\text { Aceptación } \\
\mathrm{n}(\%) \\
\end{array}$ & $\begin{array}{c}\text { Identificación } \\
\mathrm{n}(\%)\end{array}$ & $\begin{array}{c}\text { Inducción } \\
\text { a la acción } \\
\mathrm{n}(\%)\end{array}$ \\
\hline \multirow{2}{*}{$\begin{array}{l}\text { Prueba } \\
\text { piloto }\end{array}$} & $\begin{array}{c}\text { No necesita } \\
\text { reformas }\end{array}$ & $4(80)$ & $5(100)$ & $4(80)$ & $5(100)$ & $5(100)$ \\
\hline & $\begin{array}{l}\text { Necesita } \\
\text { reformas }\end{array}$ & $1(20)$ & $0(\mathrm{~N} / \mathrm{A})$ & $1(20)$ & $0(\mathrm{~N} / \mathrm{A})$ & $0(\mathrm{~N} / \mathrm{A})$ \\
\hline \multirow{2}{*}{ Poblacional } & $\begin{array}{l}\text { No necesita } \\
\text { reformas }\end{array}$ & $36(72)$ & $47(94)$ & $38(76)$ & $33(66)$ & $40(80)$ \\
\hline & $\begin{array}{l}\text { Necesita } \\
\text { reformas }\end{array}$ & $14(28)$ & $3(6)$ & $12(24)$ & $17(34)$ & $10(20)$ \\
\hline
\end{tabular}

En la validación poblacional del franelógrafo, como se observa en la tabla 3 , el $32 \%$ requiere modificaciones en la comprensión, $10 \%$ en atractividad, 36\% en aceptación, 26\% identificación y solo el $4 \%$ en inducción a la acción. En la tabla 5, se muestra el consenso de los comentarios recibidos en las variables para la mejora del material didáctico.

Tabla 5. Resultados de validación poblacional del franelógrafo

\begin{tabular}{|c|c|}
\hline $\begin{array}{c}\text { Variables de } \\
\text { validación }\end{array}$ & Comentarios \\
\hline Comprensión & $\begin{array}{l}\text { Que el lenguaje utilizado para la explicación del tema } \\
\text { sea acorde al grupo. "En la explicación se dijo algunas } \\
\text { palabras técnicas que posiblemente no todos } \\
\text { comprendan, por ejemplo: Lípidos". (P1). Es bastante } \\
\text { información y es difícil explicarlo a otras personas. }\end{array}$ \\
\hline Atractividad & El tipo de letras de los títulos no son muy llamativos. \\
\hline Aceptación & $\begin{array}{l}\text { "Hay que contextualizar los alimentos al contexto } \\
\text { rural, la piña, no hay por el pueblo." (P2) "No todos } \\
\text { entienden bien español, algunos entienden más la } \\
\text { maya." (P3) y "Debe ser más contextualizado a la } \\
\text { alimentación comunitaria." (P4) }\end{array}$ \\
\hline Identificación & $\begin{array}{l}\text { La piña no hay aquí en el pueblo, el aceite de canola y } \\
\text { de girasol no son de por aquí. }\end{array}$ \\
\hline $\begin{array}{l}\text { Inducción a la } \\
\text { acción }\end{array}$ & $\begin{array}{l}\text { No siempre se puede comprar los alimentos y se come } \\
\text { lo que hay. }\end{array}$ \\
\hline
\end{tabular}

Con respecto a la Validación poblacional del rotafolio, los resultados reflejaron que el $28 \%$ necesita mejoras en comprensión, $6 \%$ en atractividad, $24 \%$ en identificación, $34 \%$ en aceptación y 20\% en inducción a la acción (Tabla 4).

En la tabla 6, se muestra los comentarios de los participantes en cada variable de validación para la mejora del material didáctico. 


\section{Artículo Original}

Tabla 6. Resultados de validación poblacional del rotafolio

\begin{tabular}{|c|c|}
\hline $\begin{array}{c}\text { variables de } \\
\text { validación }\end{array}$ & Comentarios \\
\hline Comprensión & $\begin{array}{l}\text { Hay mucha información y no todo se logra captar bien. } \\
\text { Se entendió más o menos. Poder explicarlo a otras } \\
\text { personas está difícil, porque son varias cosas y se } \\
\text { olvida. "Las letras están un poco pequeñas y desde mi } \\
\text { lugar no se ve muy bien". (P5) }\end{array}$ \\
\hline & No se usa lenguaje que toda la población entiende. \\
\hline Atractividad & $\begin{array}{l}\text { Hay elementos innecesarios en el material, algunos } \\
\text { alimentos. El tipo y tamaño de letra no resultan muy } \\
\text { llamativos y los colores de algunas imágenes no se } \\
\text { aprecian muy bien y por lo tanto no resaltan la } \\
\text { información. }\end{array}$ \\
\hline Aceptación & $\begin{array}{l}\text { "Hay que contextualizar los alimentos al contexto } \\
\text { rural." (P6) }\end{array}$ \\
\hline Identificación & $\begin{array}{l}\text { Hay expresiones que no resultan familiar. Algunas } \\
\text { imágenes no se relacionan con la vida cotidiana. "El } \\
\text { aceite de coco, de girasol no hay aqui". (P7) }\end{array}$ \\
\hline $\begin{array}{l}\text { Inducción a la } \\
\text { acción }\end{array}$ & $\begin{array}{l}\text { Está bien lo que se dice, pero a veces no podemos } \\
\text { hacerlo, comemos lo que nos alcanza y tenemos en la } \\
\text { casa. }\end{array}$ \\
\hline
\end{tabular}

Finalmente, cuando se realizó el estadístico alfa de Cronbach, como se observa en la tabla 7 , los resultados del coeficiente muestran que el instrumento de validación del rotafolio tiene una confiabilidad aceptable, mientras que el del franelógrafo reflejó una confiabilidad buena lo que significa que los instrumentos y la información plasmada en los materiales didácticos tienen un grado de confiabilidad y validez (Chaves y Rodríguez, 2017).

Tabla 7. Resultado del coeficiente alfa de Cronbach de la validación poblacional

\begin{tabular}{lc}
\hline \multicolumn{1}{c}{ Material didáctico } & $\begin{array}{c}\text { Coeficiente de Alfa de } \\
\text { Cronbach }\end{array}$ \\
\hline Franelógrafo & 0.836 \\
Rotafolio & 0.761 \\
\hline
\end{tabular}

Fuente: Prueba de Coeficiente alfa de Cronbach.

\section{Discusión}

Los resultados obtenidos en el coeficiente de alfa de Cronbach evidencian que los instrumentos de este estudio son estadísticamente confiables, lo que brinda certeza que la información contenida en los materiales permite medir la comprensión, la atractividad aceptación, identificación e inducción a la acción sobre el tema de la alimentación saludable. Estos resultados son semejantes a los de Barradas, et al. (2020), en la cual demostraron que cinco de sus instrumentos validación eran confiables y por lo tanto sus correspondientes materiales didácticos estaban listos para poder utilizarse como están, de todos estos materiales resaltamos los resultados obtenidos en el rotafolio $(\alpha=0.81)$ y franelógrafo $(\alpha$ $=0.99$ ) de dicho estudio. Por otro lado, autores como Gómez, Cerrado y Rangel (2018), manejando las mismas variables de validación y misma escala de evaluación al del presente estudio, evidenciaron puntajes $\geq 0.9$, demostrando que el instrumento tiene confiabilidad excelente y por lo tanto resultados favorables hacia las variables de validación y hacia el material mismo. En esta misma línea Chávez y Rodríguez (2017), en la validación de un cuestionario, evidenciaron que los ítems empleados tenían un alto grado de confiabilidad.

Con base a lo anterior, se puede decir que la aplicación de la prueba de alfa de Cronbach es igual de eficiente independientemente del tipo de material e ítems formulados, de aquí la relevancia que tiene su aplicación para evaluar la confiabilidad a fin de tener una mayor objetividad, precisión y consistencia de los resultados de cada una de las variables de los instrumentos de validación.

En esta investigación, la validación técnica se realizó con la participación de profesionales de la nutrición, representando un aspecto bastante favorable, ya que permitió identificar pautas concisas de mejora, en el diseño, así como una mejor adecuación de la información, lo cual es consistente con lo que plantea Salazar, et al. (2012), al ratificar que los materiales sobre nutrición deben ser revisados por expertos en el tema, para lograr unificar criterios de forma como de contenido, para que el mensaje sea más claros y comprensibles, de igual manera las contribuciones de jueces o expertos conllevan a enriquecer, así como mejorar la aplicabilidad del material didáctico, porque permite identificar si se requiere reformular la información, sustituir términos o las imágenes empleadas, para garantizar que el material que llegue a la población sea de calidad (Moura I, et al.,2017).

Por lo que las modificaciones realizadas tras la validación técnica se vieron reflejadas positivamente en los resultados de las pruebas piloto, en los cuales tres de las variables evaluadas no necesitaron reformas, siendo solo la comprensión y aceptación los que presentan porcentajes mínimos de reforma. 


\section{Artículo Original}

En la validación poblacional se pudo observar que las características de atractividad e inducción a la acción fueron mejor calificadas, ya que un mínimo porcentaje refirió que se necesitan reformas en cuanto al tamaño y colores de letras e imágenes, mientras que la inducción a la acción los resultados evidenciaron que la población identifica que debe realizar cambios en su alimentación y estilos de vida, para prevenir enfermedades, así mismo mencionaron que su consumo de alimentos lo hacen de acuerdo a lo que tienen acceso y disponible en su comunidad. Siendo esto similar a lo obtenido por Franco, et al.(2018), en su la validación de materiales, en la cual la atractividad reflejó respuestas favorables a comparación de las otras variables, siendo necesario realizar solo mínimas modificaciones, respecto al tamaño de la letra, mientras que en la segunda variable se evidenció que a pesar de que se refuercen conductas presentes en los participantes, no todos tenían la costumbre, la disponibilidad y accesibilidad de adquirir los alimentos que se proponían en el material. De aquí que se recalca la importancia de que los materiales trasmitan información que motiven y refuercen las intenciones de cambio de conducta en alimentación y nutrición, porque la recepción del mensaje permite que los participantes puedan confrontar sus hábitos y percepciones con la información que van adquiriendo (Arismendi, et al.,2015).

Por otra parte, el rotafolio y el franelógrafo reflejaron mayor necesidad de mejoras en la comprensión, aceptación e identificación. Este resultado es parecido a lo encontrado por Barradas, et al. (2020), al demostrar que dos de sus materiales didácticos (rotafolio y memorama) reflejaron necesidad de mejoras en las variables de entendimiento e identificación, siendo estas relacionadas en cuanto a la redacción y la adecuación de las imágenes con el contexto cultural de su población de estudio, siendo similar a lo encontrado en el presente estudio, en la cual la población indicó que la explicación de los materiales no se adecuó a un lenguaje cotidiano y más comprensible, lo que se puede atribuir al hecho de que en los grupos convocados para la validación, había personas maya hablantes, por lo que al realizar la explicación en español dificultó que se comprendiera la información, lo cual difiere a lo observado en ambas pruebas piloto, en las cuales la explicación de los materiales se realizó en lengua maya, reflejándose un porcentaje muy bajo de necesidad de reformas en la comprensión, las cuales fueron referentes a la extensión de la información y palabras técnicas. Lo anterior evidencia la necesidad de emplear un lenguaje adecuado a las características de los destinatarios, así como el emplear imágenes adecuadas que resalten la información que se desea transmitir, para facilitar la comprensión del mensaje, independientemente si los participantes saben leer o no (Fernandes, Pereira, Paiva y De Souza, 2020).

De igual manera en la presente investigación se evidenció la necesidad de contextualizar los alimentos a la región, ya que la población refirió que algunos alimentos utilizados no eran propios de su comunidad. Estos resultados sin duda alguna evidencian modificaciones íntimamente relacionadas con aspectos socioculturales de la población de estudio, lo cual se correlaciona con lo que señalan Franco, et al. (2017), que los aspectos culturales y simbólicos su población de estudio, favorecía el arraigo a ciertos alimentos, así como formas de preparación artesanales y de arraigo ancestral. Resultado similar fue lo obtenido por Solano, Casas y Guarín (2019), en su investigación, en el cual la población refleja no sentirse identificada con ciertas imágenes utilizadas en el material, debido a que estas no se consideraban propias del contexto de la comunidad.

Por todo lo anterior esta investigación demuestra la importancia de someter los materiales didácticos que se pretenden utilizar en la promoción de la salud a un proceso de validación, para poder identificar las mejoras, realizar las modificaciones correspondientes y con esto poder garantizar que sean acorde a las necesidades y características de la población, para transmitir mensajes claros y de calidad, tal como lo postulan Ziemendorff y Krause (2003) y la OPS (1984).

\section{Conclusiones}

El diseño del rotafolio y el franelógrafo son estadísticamente confiables para la población adulta de la región sur de Yucatán, lo cual significa que pueden ser utilizados como recursos didácticos en la promoción de la alimentación saludable, sin embargo, para poder alcanzar niveles de confiabilidad más altos es deseable realizar las mejoras que surgieron en el proceso de validación y someter a una segunda fase de evaluación con la población para tener la versión final y así verificar 
que estén adecuados concretamente a las características, tanto culturales como socioeconómicas de la población.

\section{Bibliografía}

Arismendi, L.J., Carmona, I.C., Rodríguez, L.N., Alzate, T. (2015). Validación del juego reglado "Chefcitos", para promover hábitos de vida saludable y el consumo de frutas y verduras en escolares mayores de siete años. Perspect Nut Hum, 17 (1): 67-76.

DOI: 10.17533/udea.penh.v17n1a06

Ayuso, G., y Castillo, M.T. (2017). Globalización y nostalgia. Cambios en la alimentación de familias yucatecas. Estudios sociales ,27(50). http://www.scielo.org.mx/scielo.php?script=sci_ arttext\&pid=S0188-45572017000200004

Barradas, R., Lendechy A., Coop, F., y Pavía, E. (2021). Validación de materiales didácticos para promover la alimentación saludable en una estancia privada de adultos mayores en Mérida, Yucatán. Rev. Salud y Bienestar Social,5(2). https://www.revista.enfermeria.uady.mx/ojs/inde x.php/Salud/article/view/110/61

Chaves, E., Rodríguez, L. (2017). Análisis de confiabilidad y validez de un cuestionario sobre entornos personales de aprendizaje (PLE). Rev Ensayos Pedagógicos, XIII (1): 71-106. DOI: http://dx.doi.org/10.15359/rep.13-1.4

Díaz Brito, Y., Pérez Rivero, J. L., Báez Pupo, F., Y Conde Martín, M. (2012). Generalidades sobre promoción y educación para la salud. Revista Cubana de Medicina General Integral,28(3), 299-308.

http://scielo.sld.cu/scielo.php?script=sci_arttext \&pid=S0864-

21252012000300009\&lng=es\&tlng=es.

Fernandes, J., Pereira, I., Paiva, S.K, De Souza, A.C., Fernandes, I.K. (2020). Validación de material educativo para el cuidado de la persona con ostomía intestinal. Rev Latino-Am. Enfermagem., 28: e3269. DOI: 10.1590/1518-8345.3179.3269
Fondo de las Naciones Unidas para la Infancia. (2003). Guía metodológica y video de validación de materiales IEC.

https://www.unicef.org/peru/informes/gu\%C3\% ADa-metodol\%C3\%B3gica-videovalidaci $\% \mathrm{C} 3 \% \mathrm{~B} 3 \mathrm{n}$-de-materiales

Franco, A., Alzate, T., Granda, D.M., Hincapié, L.M., Muñoz, L.M. Validación de material educativo del programa "Niñ@s en Movimiento" para el tratamiento de la obesidad infantil. (2018). Rev. Fac. Nac. Salud Pública, 36 (3): 109-119.

DOI:

https://doi.org/10.17533/udea.rfnsp.v36n3a11

Gómez, A.L., Cerrado, R., Rangel, R.E. (2017). Validez del material educativo de un programa de educación ambiental- sanitario no formal. Educere,22(71): 1-5. https://www.redalyc.org/jatsRepo/356/35656002 011/html/index.html\#redalyc_35656002011_ref 12

Marín, A. D., Sánchez, G.; y Maza, L. L. (2014). Prevalencia de obesidad y hábitos alimentarios desde el enfoque de género: el caso de Dzutóh, Yucatán, México. Estudios sociales, 22(44), 6490.

http://www.scielo.org.mx/scielo.php?script=sci arttext\&pid=S0188-

$45572014000200003 \& \operatorname{lng}=\mathrm{es} \& \operatorname{tlng}=\mathrm{es}$.

Moura, I.H, Silva, A., Rocha, A.F., Lima, L.H., Moreira, T.M., Silva, A.R. (2017) Construcción y validación de material educativo para prevención de síndrome metabólico en adolescentes. Rev. Latino-Am Enfermagem, 25: e2934.

DOI: $10.1590 / 1518-8345.2024 .2934$

Organización Panamericana de la salud. (1984). Guía para el diseño, utilización y evaluación de materiales educativos de salud. Washington: OPS.

Salazar, A.A., Shamah, T., Escalante E.I., Jiménez A. (2012). Validación de material educativo: estrategia sobre alimentación y actividad física en escuelas mexicanas. Rev Esp Comun Salud, 3(2): 96-109. 
Solano, M.H., Casas, C., Guarín, A. (2019). Experiencia de validación de material educativo para pacientes anticoagulados en un servicio de consulta externa. Repert Med Cir, 28(2):97-104. DOI: 10.31260/RepertMedCir.v28. n1.2019.917

Vásquez, M.D. (2014). Importancia de la diversidad de materiales educativos en los rincones de aprendizaje en el nivel inicial (informe Previo a grado académico y título de Licenciada en Educación Inicial y Preprimaria). Universidad Rafael Landívar, Quetzaltenango.

World Health Organization. Division of Health Promotion, Education, and Communication. (1998) . Promoción de la salud: glosario. Organización Mundial de la Salud.

https://apps.who.int/iris/bitstream/handle/10665/ 67246/WHO_HPR_HEP_98.1_spa.pdf?sequenc $\mathrm{e}=1$ \&is Allowed $=\mathrm{y}$

Ziemendorff, S., Krause, A. (2003). Guía de validación de materiales educativos con enfoque en materiales de educación sanitaria. Chiclayo, Perú: Convenio Gtz - OPS. 